Cinémas

Revue d'études cinématographiques

Journal of Film Studies

\title{
Entre réalité et idéalité : les tracés de la voix au cinéma
}

\section{Lucie Roy}

Volume 1, numéro 3, printemps 1991

URI : https://id.erudit.org/iderudit/1001070ar

DOI : https://doi.org/10.7202/1001070ar

Aller au sommaire du numéro

\section{Éditeur(s)}

Cinémas

ISSN

1181-6945 (imprimé)

1705-6500 (numérique)

Découvrir la revue

Citer cet article

Roy, L. (1991). Entre réalité et idéalité : les tracés de la voix au cinéma. Cinémas, 1(3), 128-144. https://doi.org/10.7202/1001070ar

\section{Résumé de l'article}

Ce texte cherche plus ou moins à rendre compte du mouvement de l'écriture filmique. En interrogeant le sens de la voix et, plus particulièrement, l'intervention des silences comme tracés de la discursivité, il appert que les silences agissent comme les réseaux rayonnants de la scripturalité filmique en marquant, entre autres, la nécessité de l'écart, de l'intervalle et de l'attente. L'auteure procède à l'analyse syntaxique des silences sonores et écraniques dans le film Les Ailes du désir afin de vérifier comment l'état de veille de la réalité dans les images filmiques et l'intervention des voix qui les écarte et les traverse font réciproquement jouer le paradoxe même de l'écriture filmique : la constante traversée de l’idéel dans le réel. 


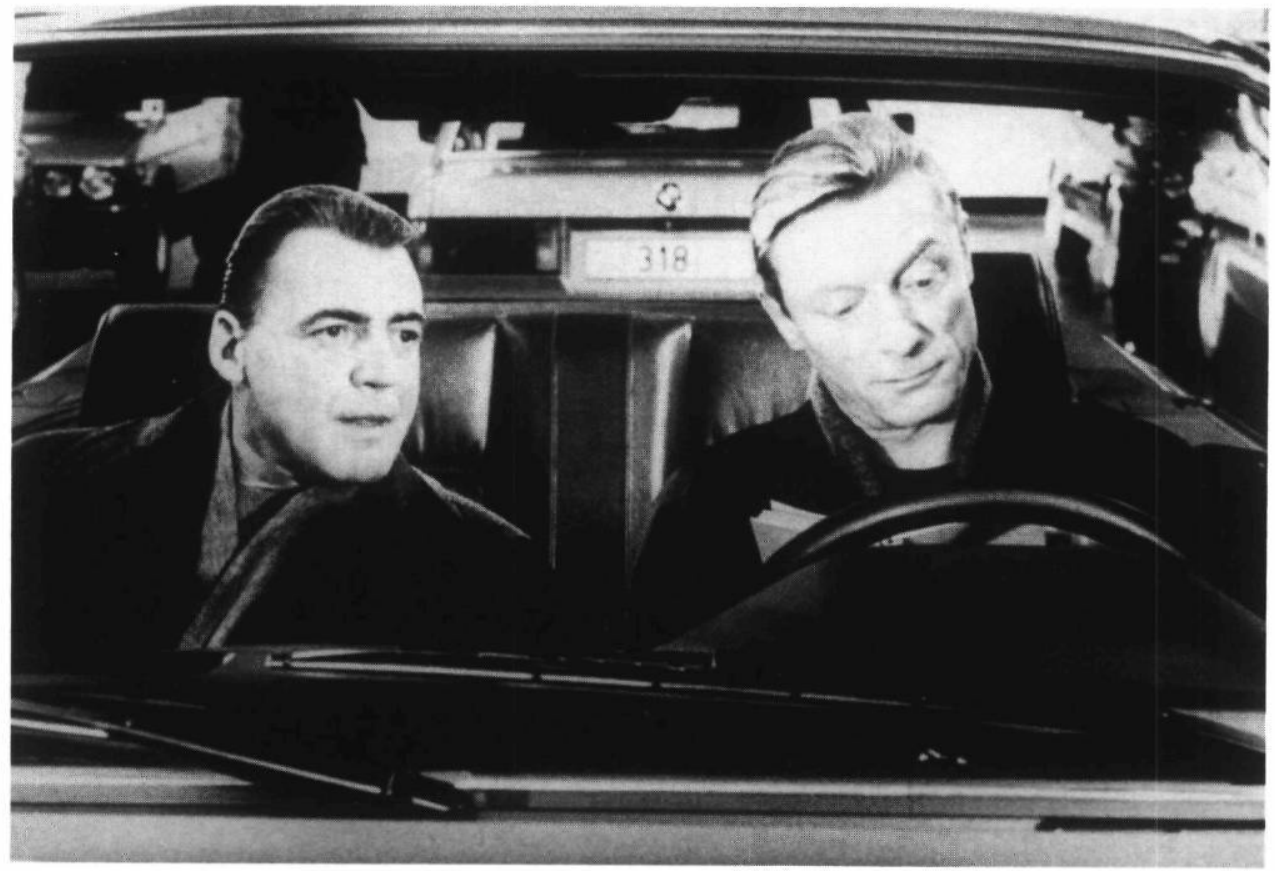

Les Ailes du désir de Wim Wenders (1987)

Coll. Cinémathèque québécoise 


\title{
Entre réalité et idéalité: les tracés de la voix au cinéma
}

\section{Lucie Roy}

\begin{abstract}
RÉSUMÉ
Ce texte cherche plus ou moins à rendre compte du mouvement de l'écriture filmique. En interrogeant le sens de la voix et, plus particulièrement, l'intervention des silences comme tracés de la discursivité, il appert que les silences agissent comme les réseaux rayonnants de la scripturalité filmique en marquant, entre autres, la nécessité de l'écart, de l'intervalle et de l'attente. L'auteure procède à l'analyse syntaxique des silences sonores et écraniques dans le film Les Ailes du désir afin de vérifier comment l'état de veille de la réalité dans les images filmiques et l'intervention des voix qui les écarte et les traverse font réciproquement jouer le paradoxe même de l'écriture filmique: la constante traversée de l'idéel dans le réel.
\end{abstract}

\begin{abstract}
This text seeks to more or less describe the evolution of filmic écriture. Focusing on the meaning of voice, and more especially on the role of silences as discourse makers, it is seen that silences act as radiating networks in filmic writing which underline the necessity for distance, delay and expectation. The author analyses the syntax of silences in the soundtrack and on the screen in the film The Wings of Desire in order to see how the state of reality expectancy in the filmic images and the voices which separate and traverse them, reciprocally bring into play the central paradox of filmic écriture: the constant crossover from the ideal to the real.
\end{abstract}


À nouveau rencontres avec des mots isolés comme: chute de pierre, durs roseaux, temps. Blanchot

Il y avait une lente pente paresseuse. Très loin derrière, une ombre blanche. J'avais le coeur qui s'étranglait. Cette ombre blanche dominait une rivière patiente à peine visible puisque des ronces l'encadraient. Le vent était puissant. Le ciel était lourd et impatient. Cette ombre blanche n'avait rien d'une berceuse. On aurait dit un visage sévère dominant un paysage-enfant pris en flagrant délit de verdure. Cette ombre avait l'allure d'une caricature à force d'imposer de hauts contrastes à cet espace. Je n'ai jamais su ce qu'il y avait derrière cette ombre.

Je restais ainsi, calme. Mon regard alourdi était une sorte d'écrin pour le soleil. Je m'enveloppais d'une sorte d'aura opaque et protectrice. Je parlais longuement, comme une enfant, de paysages inouïs que je savais habiter comme une sorcière d'une géographie planétaire. J'étais une hyène dans une forêt tropicale, une louve pour les étoiles. Pour elles, je hurlais la nuit. Elles me parlaient de la sagesse d'un temps de la pensée qui, comme un mouvement marin, arrivait à faire en sorte que je puisse parcourir les âges. J'habillais mon ventre de la paume de mes mains. Je me voyais vieille; je m'imaginais folle; je me voyais marcher dans une sorte d'étendue temporelle que je repoussais sans effort et interminablement.

J'étais restée allongée et lorsque le temps fût venu, j'ai fait la danse des enfants, j'ai soulevé la poussière: magie, alchimie. Je refaisais ma jeunesse au parfum de l'atmosphère. J'étais douce, immatérielle, transparente comme une sorte de mélopée sans visage. Indicible, je me suis tue.

J'avais la tête à la fois lourde et légère. J'aurais voulu tout raconter. Qu'est-ce que je faisais ici? Je faisais la grève de la faim, je faisais la grève de l'amour. "Je faisais une marche juive en un exode sans terre, je n'en aurais jamais eue; je faisais une marche juive en un exode sans corps, car on me l'aura pris ${ }^{l_{\text {.» }}}$

Je parsemais mes rêves de longues pierres plates, je marchais dessus les pieds nus. Je chantais des hymnes millénaires. Il me fallait appeler les horizons et les ramener vers moi afin que je puisse m'abriter en eux comme en de douces couvertures. J'insultais les âges au nom de la misère humaine. J'étais une prêtresse de la paix.

Il y avait des incantations. Un feu. Une pierre. Un creuset. Le même ciel lourd et une ombre blanche. Des rochers taillés. 
La marche des Juifs. Des pierres. J'avais du sable dans les mains. C'était la nuit. Je réinventais l'architecture, la géométrie et la musique.

J'étais sauvage, je réinventais des silences.

La tête me tourne. J'ai les jambes comme de longs lambeaux de chair. Je me sens désuète et idiote. J'ai rêvé, je crois.

Je m'étais laissée enivrer par une sorte de vertige: j'avais franchi les frontières de la réalité, j'avais appris à conjuguer les espaces, les temps, les ombres, les lumières et j'y avais entendu des paroles sans voix.

\section{L'écriture}

Je ne sais pas de façon précise d'où vient l'écriture: de quel désir elle émane ni de quelle volonté elle se nourrit. Je ne sais pas, au plan de la connaissance, ce qu'elle réussit véritablement à combler. Je ne sais pas davantage jusqu'à quel point l'écriture cinématographique peut être comparable à la rêverie ou au rêve: à cette sorte d'ubiquité qui, quoique inconsciente dans le rêve, utilise plusieurs lieux et une certaine multiplicité des temps. Je ne sais pas si le cinéma est né du désir de rêver ou, plus simplement encore, de cette obsession de franchir ou plutôt de se ressaisir de l'espace et du temps et de les élever en tracés formels de la pensée. Je ne sais pas précisément de quelle urgence vient le cinéma ni jusqu'à quel point celui-ci est le lieu de la re-présentation des représentations comme œuvre du penser, du voir et de l'entendre scripturalisés dans le film.

Je sais pourtant que chacune des mises en images des pans de la réalité, chacune des mises en ombre ou en lumière des objets, des personnages ou des lieux contenus dans les images filmiques, chacune des mises en ombre ou en lumière de la voix au cinéma appelle la réalité en la transformant au même moment en idéalité en les forçant ainsi à appartenir au réseau phénoménal de l'écriture.

"À nouveau rencontres» au cinéma avec des mots et des images isolés, conjugués et, davantage, enchevêtrés par les temps du récit, toujours multiples, toujours lacérés et enchevêtrés par la profusion des espaces qui se donnent à lire dans le film comme "chute de pierre, durs roseaux». "À nouveau rencontres» avec l'essor scripturalisant des images et, davantage, des silences qui agissent parfois comme les noyaux rayonnants de la mise en présence de la pensée et, davantage, comme la mise en présence du temps de la pensée à travers l'empreinte des temps du récit ou de la fiction au cinéma.

Je me propose d'aborder ici l'étude de la voix en considérant l'intervention des silences au cinéma comme des «moments ou 
des monuments ${ }^{2}$ de la signification en vertu de l'opération de l'écriture filmique ou, en d'autres termes, en vertu du passage du réel à l'idéel dans le film.

Pour ce faire, je m'attacherai d'abord à définir les silences sonores et écraniques au cinéma, à vérifier sommairement l'importance de leurs fonctions syntagmatiques ou, plutôt, de leurs fonctions syntaxiques, je décrirai certaines séquences du film Les Ailes du désir de Wim Wenders pour enfin pouvoir interroger certains des enjeux scripturaux du cinéma.

\section{De la voix}

(...) non-dite, la parole est aussi faite de non-dit. Ou plutôt le dit cache ce qu'il y a à dire parfois, et parfois révèle le non-dit. La parole n'est jamais de l'ordre de l'évidence. Elle est sans cesse un jeu de voilement et de dévoilement (Ellul, p. 21).

Il est relativement facile d'admettre que le silence peut être un choix dans l'étendue des paroles à prononcer, que certaines d'entre elles agissent comme des sortes de caches pour l'esprit ou qu'elles peuvent être représentatives d'une aversion en regard de la charge d'ambiguïté qu'impose le silence; qu'au plan de la communication, celui-ci peut se poser entre le parleur et l'écoutant dans l'instant de l'attente du dire et de l'écoute de même que dans l'interstice compris entre la pensée et la parole se faisant discours. Il est enfin facile d'admettre que le silence peut être la trace ou, indistinctement ici, l'indice d'une accalmie satisfaite de la parole ou, plus simplement, la marque de la clôture de la parole.

Il appert pourtant, qu'au plan de la signification ou de la connaissance, le silence peut être l'empreinte d'une nostalgie, d'une alerte, d'un refus, d'une quête, d'une enquête ou d'une entente muette.

Le sentiment qu'il a, chaque fois qu'il entre et lorsqu'il prend connaissance de l'homme âgé, robuste et courtois, qui lui dit d'entrer, se levant et lui ouvrant la porte, c'est que l'entretien est commencé depuis longtemps (Blanchot, 1983, p. IX).

En tant déjà que halo émotif, qu'instrument d'une sorte de lenteur énonciative; en tant qu'envers de la voix, le silence suffit parfois à exprimer la complexité de certaines pensées, les hauts cris de certaines émotions, leur ambiguïté et, parfois, leur charge vertigineuse.

Le silence fait donc partie du discours, de l'avant, de l'après et des moments du discours, malgré le fait qu'il peut exprimer la 
plaine de l'imprononcé en s'élevant ainsi et de façon paradoxale en indice de l'indicible.

Bien que je ne crois pas utile de chercher à définir longuement le silence comme signe, voire, comme négation de signe, songeant à une part de la théorie sociolinguistique du signe, il nous faut admettre que certains silences ont acquis un tel degré d'arbitraire, qu'ils peuvent, par exemple, nous sommer de comprendre la gravité ou la réprobation. Ils sont, en somme, représentatifs d'une expression pour la pensée. Les silences peuvent se substituer aux mots et tenir lieu d'objet de représentation pour la pensée, malgré le fait qu'il donne nécessairement à lire un signifiant mis en rapport ou en opposition avec d'autres signifiants, visuels ou linguistiques, en affirmant ainsi sa différence et sa volonté énonciative et en décrétant le paradoxal de sa substantialité, c'est-à-dire la quasi-absence ou l'immatérialité de son signifiant et, finalement, ses possibles significations.

Pour reprendre plus ou moins certains aspects théoriques proposés par Derrida et auxquels Marie-Claire Ropars a consacré une étude importante du cinéma relativement aux notions de trace et de différence, en niant d'une certaine façon la verticalité de la signification (SA/SÉ) et en irradiant ou en niant l'horizontalité de l'énonciation (les articulations et la syntaxe), le silence commande l'examen du procès des différences des signes et de leurs traces au sein de l'annonciation de la voix.

En clair, en détournant la dyade signifiant/signifié, en élaguant le principe des unités de première et de deuxième articulations, en lacérant l'ordre de l'énonciation par des intervalles ou des interruptions, le silence nous incite à penser la différence comme structure et comme sens, et à repenser le non absolu des paroles et des signes qui sont supposés l'énoncer. Les intervalles silencieux conduisent à penser le silence comme tracé discursif ou comme tracé de la discursivité. En somme, certains silences sont en eux-mêmes représentatifs de la parole à exprimer et ils sont constamment représentatifs des enjeux mêmes de l'écriture.

Tracement, écartement, ces termes supposent une conception dynamique, mais non linéaire, des rapports entre les éléments écartés spatialement. C'est cet écartement qui saisit et traverse le signe, le renvoyant par sa différence avec un autre signe, à la trace qui le brise, en barrant toute référence à un sens originel. Le concept de trace, substitué à celui du signe, organise le démantèlement du sens (...) il n'y a pas d'origine absolue du sens en général ${ }^{3}$.

Il appert que le silence intervient d'une certaine façon constamment dans l'ordre de l'énoncé en interrogeant les signes qui le constituent, en imposant l'examen de la présence-absence, du voilement-dévoilement de la parole, des traces et des inter- 
valles qui la constituent et en imposant un relatif décryptage du temps silencieux de l'énonciation. À la limite, il incite à songer à une sorte d'immanence de sens par la mise en évidence du scripteur plutôt que par celle de l'énoncé ou, à tout le moins, il exhorte l'écoutant à prendre en compte la quête de la pensée au sein de la parole.

En d'autres termes, l'intervention du silence dans la parole force à considérer la chute des mots et certaines de leurs impossibilités sémantiques, à repenser la présence du paradigme dans le syntagme: le constant rapport de la notion du choix énonciatif au sein de l'ordre syntaxique.

\section{De la voix au cinéma}

L'obsession des yeux désigne autre chose que le visible. Blanchot, 1984 , p. 21.

Je ne crois pas qu'il faille aborder l'étude du silence au cinéma sous le regard d'une analyse exclusive de la voix. L'étendue des outils de la signification au cinéma ne pourrait, en effet, s'y résoudre. Car, s'il faut considérer le silence comme l'étude d'une certaine parole, il faut aussi considérer son intervention au cinéma comme la pratique d'une certaine écriture en prenant en compte l'intervention des silences dans les bruits, les musiques et les voix de même qu'entre les images et les voix.

Cela dit, il se peut que le récit soit pris en charge par les voix et les silences, et que les images agissent comme des sortes de réquisitions temporelles. Dans ce cas-ci, le silence, par opposition au déroulement de la bande-image ou, pour ainsi dire, par opposition au manifeste de l'obsession des yeux dans le film, chercherait à s'assurer de la manifestation de l'invisible: la mise en présence du temps de la pensée comme idéalité et scripturalité.

L'étude du silence au cinéma impose l'examen même de l'écriture filmique, puisque lorsque le silence ou l'envers de la voix se fait écriture, il force à prendre en considération le tissu formel et temporel sur lequel il est déposé et il incite à vérifier ce qu'il rompt, irradie ou dynamise.

Il appert, en effet, qu'au cinéma comme ailleurs, le silence agit comme tracement de la voix, comme tracé de sens, comme écart entre la mise en voix et le silence, comme tracé discursif, comme une sorte de présence entre les mots, les images ou dans les images mêmes; entre les plans et les séquences, entre les images et les voix. Le silence fait partie du champ scriptural du 
cinéma dans le mesure où il participe évidemment à l'opération du «penser» dans la configuration même des images de la réalité de même que dans la refiguration de la pensée se faisant discours et écriture.

À cet égard, il semble impossible de parler de la scripturalité cinématographique en vertu du passage de la réalité à l'idéalité ou en vertu de la refiguration de la réalité dans le film sans prendre en considération l'entrelacement de ces modales énonciatives que sont les voix, les bruits, les musiques, les images et les silences qui représentent en eux-mêmes le tissu phénoménal de la réalité et, au cinéma, le réseau phénoménal de la scripturalité.

\section{Lorsque l'image se fait voix}

Il a fallu une grande imagination scientifique pour comprendre que ce ne sont ni les charges ni les particules mais le champ de l'espace entre les charges et les particules qui est essentiel pour la description des phénomènes physiques ${ }^{4}$.

Par tradition, la quasi-absence de définition du silence avait imposé la prémisse initiale généralement acceptée qui voulait que celui-ci corresponde à une sorte d'étendue de la parole comme telle inqualifiée et dénuée de sens. Cherchant à trouver une certaine correspondance entre le silence de la parole et celui de l'espace écranique, je ne pouvais que reconnaître les espaces vides contenus dans les images de même qu'entre les plans et les séquences.

Je ne crois pas avoir à développer davantage cette assertion qui veut que le vide à l'écran ou le silence écranique structure et oriente la lecture des objets contenus dans l'image et, qu'en ce sens, il est représentatif d'une sorte de «loi» de l'entour ou du pourtour ${ }^{5}$. C'est, en effet, par une sorte d'émergence spatiale ou de contraction du vide - qu'il soit coloré ou non - , que les objets, les personnages ou les paysages se donnent à lire dans l'image.

À cet égard, nous pouvons d'ores et déjà admettre que le silence écranique peut être considéré comme une fonction structurelle en vertu de la mise en présence des objets contenus dans l'image qu'il permet et que les silences ou les intervalles écraniquement silencieux épanchés dans la continuité filmique (les fondus, les volets, les noirs, les blancs, même plus ou moins accentués) ont une fonction temporelle en vertu des interruptions qu'ils creusent entre les plans et les séquences en marquant ainsi le passage d'un temps à un autre. Plus encore, les silences au cinéma peuvent être compris comme des interstices ou des écarts 
posés entre les voix et les images ou entre les images elles-mêmes en créant ainsi une sorte de tension entre les systèmes narratifs.

Prise dans un autre sens, l'étude de l'utilisation de l'espace et du temps au cinéma nous amène à dire que là où il $\mathrm{y}$ a une coupure silencieuse dans la continuité cinématographique, structurante par son étendue ou sa fonction différentielle, il y a écriture.

Le silence au cinéma, les ruptures qu'il fabrique au sein de la diégèse peuvent, en somme, constituer les noyaux irradiants de l'écriture filmique dans la mesure où ils conduisent, entre autres, à temporaliser la syntaxe filmique et la continuité diégétique.

Le silence, en tant que lieu de l'interstice, en tant que lieu de l'envers de la voix, lieu de non-parole plutôt que de non-langage, en interrompant ou en faisant imploser la course de l'énonciation de la parole, du film ou de la parole contenue dans le film, interroge parfois ce mouvement associé à un autre mouvement: l'attente d'un regard à nouveau porté sur la parole ou sur le récit qui est en elle-même plus vaste en vertu de l'appel de l'écriture ou en vertu de l'appel au réseau phénoménal de l'écriture que cette attente impose.

Le silence peut contribuer à mettre en présence la spatialisation de l'espace représenté et le temps de cette spatialisation tout comme il met en présence le temps de la parole. Il interroge constamment les possibilités sémantiques et il met inévitablement en exergue le temps de la scripturalité ou les procédés temporels de la scripturalité filmique qui comprennent sommairement au cinéma le temps de la quotidienneté et le temps de la pensée.

\section{L'intervention syntaxique}

J'aimerais préciser que le silence au cinéma ne saurait de part en part être associé à un syntagme — pas plus d'ailleurs qu'il ne pouvait être associé de part en part au signe notamment en vertu de l'impossibilité d'y identifier des unités de première et de deuxième articulations - , dans la mesure où le silence n'emporte évidemment pas avec lui un alignement de morphèmes comparable à la définition linguistique de ce terme. Pourtant, puisque le silence relève de façon, disons, ontologique d'une séquence énonciative cette fois-ci temporelle, puisqu'il est inévitablement employé en rapport avec d'autres éléments filmiques, nous pouvons admettre sommairement que certains silences ont les mêmes volontés sémantiques ou, sur le plan du signifiant, des charges syntaxiques semblables. Il nous faut surtout admettre que puisque le film utilise plusieurs matières de l'expression, le silence peut faire partie et fait partie d'un syntagme intercodique. Soit il appartient à la bande sonore et il est mis en opposition à la 
bande-image du récit, soit encore en tant que silence écranique, il est mis en opposition à la voix. Je souhaite donc analyser le silence en vertu de la multiplicité des codes que le cinéma autorise.

Il appert, en effet, que certains silences relèvent de la syntaxe du film et concourent à l'énonciation des syntagmes filmiques. Cette remarque est d'autant plus probante lorsqu'il s'agit de reconnaître que les ruptures silencieuses posées dans la course filmique rompent l'ordre séquentiel et imposent des syntagmes aséquentiels ou des commutations a-séquentielles dans le film.

En termes sémiologiques, il importe donc de constater que, sur les axes paradigmatique et syntagmatique du film, il y a des voix et des silences, des vides et des pleins, tout comme il y a des syntagmes composés de silences qui appartiennent à un segment narratif (syntagmes intraséquentiels), qui se situent entre les plans, les séquences ou les segments filmiques (syntagmes interséquentiels), soit encore, ils recouvrent un ensemble des segments filmiques jusqu'à détrousser l'ordre de l'énonciation filmique (syntagmes paraséquentiels). En somme, le silence peut être faiblement marqué par rapport aux éléments filmiques coprésents ou, à l'inverse, il peut être fortement marqué et agir comme une structure de grand ou de très grand ensemble.

À l'intérieur des modalités séquentielles que je viens de décrire, ces syntagmes ou, mieux, ces sortes de morpho-syntagmes pourraient avoir plusieurs fonctions.

Le syntagme de silence objectif - que je persiste à appeler ainsi malgré son haut degré de contextualité ou d'intercodicité en brisant la course narrative du récit, a pour but de créer une sorte de temps d'arrêt, de distanciation par rapport à l'action dramatique en cours; le syntagme rythmique est, lui, il va sans dire, essentiellement formel et a pour but de s'opposer à la durée des plans ou des séquences; le syntagme d'articulation formelle sert à joindre deux plans ou deux séquences; le syntagme d'articulation diégétique a, comme son nom l'indique, un caractère plus narratif dans la mesure où il a pour fonction de joindre deux segments ou deux grands moments du récit présentés comme étant disjoints; le syntagme d'accent tonique s'oppose au syntagme de silence objectif, en créant une attente, en relançant ainsi l'action et en faisant en sorte que le spectateur anticipe et surqualifie la dramaticité des événements; le syntagme de silence psychologique peut agir comme un instrument d'énonciation de la pensée du personnage, voire, du narrateur; le syntagme de silence poétique, plus complexe que les précédents, agit comme un outil de symbolisation par renvoi à un système narratif coprésent à l'intérieur de la même diégèse. Il agit comme une pause narrative, comme un écart qui souligne sans cesse le concept discur- 
sif employé. Aussi, le fait de ponctuer l'enchevêtrement de deux circuits narratifs suffit à charger le récit d'une sorte de volonté connotative. Enfin, le syntagme a-diégétique impose des ruptures spatio-temporelles de taille dans le récit et agit en tant que négation de l'alignement ou de l'ordre séquentiel de sorte qu'il retourne la fiction au temps de son énonciation et aux procédés formels et narratifs du fait filmique.

\section{L'irradiation de la voix et des silences dans Les Ailes du désir}

La vie sous le soleil n'est-elle rien d'autre qu'un rêve? Ce que je vois, ce que j'entends, ce que je sens, n'est-ce pas simplement l'apparence d'un monde devant le monde 6 ?

"À nouveau rencontres avec des sons, [des images], des mots comme» la musique lancinante d'un violon, celle d'un violoncelle, la vue du ciel, d'un œil ou d'une ville qu'un regard survole, le son d'une harpe, la vision d'un ange avec des ailes, debout, sur la corniche d'un clocher. Le regard de l'ange surplombe la rue. L'image obscure du clocher se dessine sur le fond du ciel. Dans la rue, il y a des passants. Des enfants dans un autobus aperçoivent l'ange. Une multitude de voix se font entendre dont on ne distingue pas les paroles. Une aile s'agite et passe devant l'écran. Le son d'une harpe: le regard de l'ange descend jusque dans la rue où un homme portant un enfant marche.

La joie de lever la tête vers la lumière, ici, au grand air, la joie de voir dans les yeux des hommes les couleurs illuminées par le soleil.

L'histoire du film Les Ailes $d u$ désir raconte l'errance «planétaire» d'une sorte d'ange et elle raconte l'errance, la nostalgie et l'incertitude quotidiennes des hommes. Berlin, la ville est grise et lourde de son passé. L'ange, déjà habité par la volonté d'«avoir la sensation en marchant que toute sa charpente avance à chaque pas (...)", rencontre une femme trapéziste dont il tombe amoureux. Finalement, gagnant le monde des hommes, il la rejoint. Comme pour faire se matérialiser le temps et l'espace, les images du film, jusque-là en noir et blanc sont en couleur.

Afin de pouvoir rendre compte des procédés empruntés et de l'essor scripturalisant des mises en écarts de la voix par rapport à l'image, je m'attacherai à décrire abondamment certaines sé- 
quences filmiques qui rendent elles-mêmes compte de cette sorte de contamination du réflexif dans le filmique.

Dans ce film, la bande sonore est hantée par les voix off des hommes que cet ange entend. Les voix sémantisent les images ou, au contraire, se font murmures. Les plans sont présentés dans le film comme des systèmes isolés: le ciel, un oiseau, une femme et un enfant roulant à bicyclette, un avion. À l'intérieur de celui-ci, des passagers se parlent à eux-mêmes, la voix d'une enfant offre à l'ange de lui dessiner une charrette avec du foin. Un réalisateur cherche à mieux comprendre un personnage. Une hôtesse de l'air s'adresse à la fillette.

La musique d'un violoncelle, des chants, un regard errent longuement dans le ciel. Une tour. Des voix se confondent comme des ondes de radio qui se brouillent. Des plans aériens : une ville, des autoroutes, des édifices à logements.

La musique d'un violoncelle parcourt gravement les plans suivants: une femme âgée dans un appartement: «Trébuchez sur vos couleurs et vous n'êtes jamais à l'heure (...)». Une jeune femme cherche l'endroit où placer son frigo. Un homme entre dans l'appartement de sa mère récemment décédée. Une femme fume une cigarette. Des murs. Des enfants courent dans la ruelle. Une fenêtre. Un homme assis sur le bord de son lit: «Elle ne t'aime pas, elle ne t'a jamais aimé (...)». Un mur. Un homme: «Mon dieu, quel va être l'avenir de ce garçon (...)». Une femme dans une cuisine: «C'est pas surprenant, en dehors du rock and roll, il n'a rien appris d'autre (...)».

Fondu au noir. Un mur. Un escalier. Fondu au noir. Des voix d'enfants qui jouent se font entendre. Sous-jacente à tout cela, une voix off, celle de l'ange: "Lorsque l'enfant était enfant (...)». Des enfants jouent. L'ange se tient à l'écart et regarde. Une mère habille une enfant handicapée. «Quand a commencé le temps et où finit l'espace (...)». L'ange. Le visage de l'enfant. L'ange. L'enfant handicapée sourit à l'ange, les jouets de celle-ci sont minutieusement alignés dans la pièce. «Comment se fait-il que moi qui suis moi, avant que je devienne, je n'étais pas (...)».

Une fenêtre. Des autoroutes. Le son d'une sirène. Une femme accouche. Une ambulance. Une automobile passe. À l'intérieur, une femme frappe un homme: «Salop (...)». Dans une autre automobile, une femme âgée, accompagnée par son chien, cherche un certain cimetière. Les membres d'une famille algérienne discutent.

Un reflet de lumière sur une vitre. Un long silence. L'ange est assis dans une voiture en compagnie d'un autre ange. Un silence. Un reflet. Ils se font part des observations qu'ils ont pu recueillir: «Une passante qui a fermé d'un coup sec son para- 
pluie, s'est laissée tremper. Un écolier qui décrivait gravement à son maître comment les fougères sortaient de terre. L'instituteur sidéré (...)».

Le film Les Ailes du désir ne fonctionne pas comme un lieu fictionnel habituel. Ici, les personnages n'agissent pas comme des porteurs d'idées et d'actions pris dans un conflit qu'ils auraient eu à résoudre. Le film tend plutôt à rendre compte des temps de la pensée, celui, entre autres, d'un esprit, témoin des pensées quotidiennes des personnages qui, tels des passants diégétiques ou des figurants de la diégèse, livrent leurs inquiétudes, leur quêtes ou leurs ressouvenirs. Le circuit filmique conjugue des espaces fragmentés et a-temporalisés par l'irradiation des voix, celles des anges et celles des passants et par le constant passage d'un temps à un autre, d'un réseau scriptural à un autre.

Un métro file à toute vitesse, un homme s'inquiète de la santé d'une femme. Une femme se demande comment prier. Une autre s'inquiète des petites. Un homme se demande pourquoi il vit. Une femme âgée se demande comment elle pourra vivre avec sa petite pension. Un homme se plaint de se lever trop tôt et de ne pas trouver de travail. Un autre homme désespéré reprend soudain confiance en lui. Le métro file. Des enfants tentent d'attraper de la monnaie à l'intérieur d'un puits d'égouttement. Dans une ruelle, des enfants jouent. Un cirque. Un éléphant. Une femme trapéziste s'entraîne. L'ange la regarde. Sous le regard de cet ange fasciné, quelques images sont en couleur. Un homme annonce la fermeture imminente du cirque. Désespérée, la trapéziste songe à la fin de sa carrière, à la solitude, à ses désirs, à ses peurs. Dans sa roulotte: «Que fautil faire? (...). L'angoisse qui me rend malade (...). Comment dois-je vivre? (...). Je pense souvent de façon si fausse, je pense comme si je parlais en même temps à quelqu'un d'autre (...). À l'intérieur des yeux fermés, fermez encore les yeux. Alors même les pierres se mettent à vivre (...).» L'ange la regarde, examine des photographies épinglées sur le mur et prend une pierre, immatérielle. «(...) les couleurs, les néons dans le ciel du soir, le métro aérien rouge et jaune (...).» L'ange tente de la toucher. "Nostalgie, nostalgie d'une vague d'amour (...).» Les plans de la fin de cette séquence sont en couleur.

Silence. Fondu. Plans en noir et blanc. Une route. À l'intersection, un homme, un motocycliste est sur le point de mourir. La voix d'une passante. L'ange s'approche et prend la tête de l'homme dans ses mains. La voix de l'ange et celle de l'homme se confondent. «(...) la croix du sud (...) les plaines du Farwest, le grand lac Victoria (...) Albert Camus (...) la lumière 
de l'aurore (...).» L'ange s'éloigne, son regard surplombe des voies ferrées. «Les nervures des feuilles (...).» Un train passe. «Le rêve de la maison dans la maison, quelqu'un qui dort dans la pièce voisine, la paix du dimanche, l'horizon, la lumière de la chambre dans le jardin.» L'ange assis sur l'épaule de la statue d'un ange surplombe la ville. «(...) mon père — le ciel - ma mère, ma femme, mon enfant.»

Plus encore, les lieux : une bibliothèque, des voies ferrées, une gare, un terrain vague, un plateau de tournage agissent comme des motifs diégétiques qui évoquent l'histoire et les atrocités de guerre dont ces lieux ont été, pour ainsi dire, les témoins.

«C'en est fini du grand souffle de jadis [dans une bibliothèque, un vieil homme feuillette un livre], du va et vient à travers les siècles. Désormais, je ne peux plus penser qu'au jour le jour, mais on ne sent plus les guerriers et les rois.» Des rues dévastées, des morts ensevelis sous les décombres, des soldats. «Les choses de la paix sont toutes égales entre elles. [Un bébé mort. Des enfants morts.] Les oignons qui sèchent valent le tronc d'arbre qui traverse le marécage [un vieil homme regarde un livre], mais nul n'a encore réussi à chanter une épopée de la paix. Pourquoi la paix n'a-t-elle rien d'exaltant à la longue et pourquoi est-il si difficile de la raconter? Faut-il que je renonce? Si je renonce, l'humanité perdra son conteur. Et si jamais l'humanité perd son conteur, elle perd aussi son enfance.»

Dans le film Les Ailes du désir les silences sonores et écraniques s'insinuent entre les paroles, entre les voix et les musiques, dans les images de même que dans les interstices compris entre les plans et les séquences.

Les silences agissent principalement comme des syntagmes d'articulation formelle et, souvent à la fois, comme des syntagmes d'articulation diégétique, dans la mesure où la plupart des segments filmiques sont présentés dans le film comme étant disjoints ou non liés par une trame fictionnelle. Ils sont également utilisés comme des syntagmes psychologique et poétique. Bien qu'en voix off, les silences font partie du discours de la voix et sont évidemment représentatifs de la psychologie des personnages qu'elle soit peu ou pas développée ou qu'elle soit prise en charge par un narrateur diégétique. Davantage encore, les silences présents dans le film renvoient constamment au système narratif coprésent, c'est-à-dire à la mise en présence de la voix et, plus particulièrement ici, de la voix off, comme tracé du récit.

Utilisés dans un haut degré d'intercodicité, les silences sonores et écraniques servent d'articulation ou, mieux, de mise en écart des plans et des séquences, entre les musiques et les voix, et ils 
agissent surtout comme relais de la scripturalité du film en relançant ainsi l'écriture de la voix dans le film.

Le film Les Ailes du désir est construit sur le mode des tensions entre la voix et sa mise en abîme par le «off», entre le littéraire et le filmique, entre l'immatérialité de la pensée et la relative matérialité de l'espace, entre la mobilité et la fixité, entre le noir, le blanc et la couleur, entre la véracité des personnages, leur éphémérité ou leur absence, entre le procès de la sensation d'immanence de la réalité dans le film et la mise en présence de la scripturalité au sein même du circuit filmique.

Les voix subissent dans ce film une sorte de double détournement énonciatif dans la mesure où les personnages ne semblent pas les porter et dans la mesure où elles sont surprises ou prises en charge par ce narrateur omniprésent qu'est le personnage de l'ange.

L'espace de la réalité se trouve appelé, fouillé, ceintré dans le cadre de l'image par la quasi constante mobilité de la caméra qui abolit par là même l'appel au hors-champ. Par ailleurs, la mouvance de la caméra force l'image à subir par endroits le joug de l'asymétrie en donnant ainsi à lire des espaces vides ou des silences écraniques: réseaux structurants de la mise en présence des objets contenus dans l'image. Ce n'est pourtant pas ici la fixité des plans qui s'oppose à la mobilité de la caméra mais, à un autre niveau, la fixation des plans ou des plans-séquences qui agissent comme des collages de lambeaux d'existence qui se font empreintes des moments de monde.

De plus, l'utilisation du noir et du blanc, presque constante dans le film, semble vouloir mettre en opposition l'immatérialité de la pensée et, par code implicite et de façon paradoxale, l'aspect photographié ou documentarisé de la matérialité ou, plutôt, de la mise en matière du temps et de l'espace de la quotidienneté. Par rapport à une ontologie du cinéma, en jouant inévitablement le jeu de la préhension des parts de la réalité, en référant à la réalité comme imprégnant et empreinte, en lui imposant le noir et le blanc, en détournant l'image pour privilégier la voix, en détournant la voix pour privilégier la voix off, en imposant l'hégémonie de la coupure dans le film, Les Ailes du désir impose la lecture du réflexif dans le filmique ou, en d'autres termes, de l'idéel dans le réel. Le film ne cesse, en effet, d'affirmer la présence du perçu ou du $v u$ et, au même moment, du percevant ou du voyant. L'irradiation des voix et des silences écraniques dans les espaces filmiques incite à prendre en considération le détournement de la fiction, le tracement de la scriptu- 
ralité et les limites de la discursivité filmique par la mise en présence de la pensée d'un scripteur même fictif.

De plus, l'intervention constante de la voix et la présence des silences dans le film incitent à penser le littéraire dans le filmique ou, à tout le moins, le rayonnement sémantique et diégétique de la voix et donc, à interroger la scripturalité et le sens du temps et de l'espace au cinéma. Le tracement de la voix off apparaît ici semblable à l'utilisation du noir et blanc. L'un et l'autre impliquent les positions scripturales et spectatorielles du ressouvenir ou du soupçon d'un temps plus vaste, celui de l'histoire et celui de la pensée, qui enveloppe celui d'une quasiquotidienneté, disons, filmique.

Le silence suffit à mettre en évidence le réseau de l'écriture: la nécessité de l'intervention, celle de l'écart, celle de l'intervalle, celle de la découpe et celle de l'attente.

Université Laval

\section{NOTES}

1 Cette dernière phrase est inspirée des propos sur l'œuvre filmique Nathalie Granger dans Marguerite Duras, xuvres cinématographiques (Paris: ministère des Relations extérieures, bureau d'Animation) p. 58. En parlant des Juifs, Marguerite Duras souligne : «On ne pouvait pas prendre leur terre, ils n'en avaient pas. Alors, faute de matériaux à prendre, on s'est acharné sur leur corps.» La nouvelle proposée en introduction de cet article est évidemment de moi.

2 Cette expression est issue de ma thèse de doctorat Le Silence au cinéma ou les structures de l'inexistant (Québec: Université Laval, 1989) p. 2.

3 Jacques Derrida cité dans Ropars-Wuilleumier, Le Texte divisé (Paris: P.U.F., 1981) p. 23.

4 Einstein cité par Jakobson, Essais de linguistique générale. Les fonctions du langage. Tome 1 (Paris: Minuit, 1963) p. 139.

5 L'ensemble de ces réflexions et, plus particulièrement, l'étude des «lois» du pourtour et de l'entour à l'image, l'analyse des fonctions syntagmatiques ainsi que des intervalles diégétiques des silences sonores et écraniques au cinéma ont fait l'objet d'une étude suffisamment approfondie lors de la recherche et de la rédaction de ma thèse de maîtrise, Le Silence au cinéma ou les structures de l'inexistant 1(Québec: Université Laval, 1984). Ce mémoire a été le creuset de réflexions d'ordre sémiologique sur le silence au cinéma. À l'intérieur de ma thèse de doctorat Le Silence au cinéma ou les structures de l'inexistant 2, j'ai pu aborder l'étude psychanalytique et phénoménologique du silence au cinéma et, plus largement encore, l'étude de l'écriture cinématographique.

6 Les phrases suivantes sont tirées du film Les Ailes du désir de Wim Wenders. 


\section{OUVRAGES CITÉS}

Blanchot, Maurice. Le Dernier à parler. Paris: fata morgan, 1984.

Blanchot, Maurice. L'Entretien infini. Paris: Gallimard, 1983.

Ellul, Jacques. La Parole humiliée. Paris: Seuil, 1981. 